\title{
Influence of thermal diffusion on the laser ablation of thin polymer
} films

\author{
Journal Article \\ Author(s): \\ Fardel, R.; Nagel, M.; Lippert, Thomas; Nüesch, F.; Wokaun, Alexander; Luk'yanchuk, B.S. \\ Publication date: \\ 2008 \\ Permanent link: \\ https://doi.org/10.3929/ethz-b-000012726 \\ Rights / license: \\ In Copyright - Non-Commercial Use Permitted \\ Originally published in: \\ Applied Physics A 90(4), https://doi.org/10.1007/s00339-007-4334-9
}




\author{
R. FARDEL ${ }^{1,2}$ \\ M. NAGEL ${ }^{1}$ \\ T. LIPPERT ${ }^{2, \infty}$ \\ F. NÜESCH ${ }^{1}$ \\ A. WOKAUN ${ }^{2}$ \\ B.S. LUK'YANCHUK ${ }^{3}$
}

\title{
Influence of thermal diffusion on the laser ablation of thin polymer films
}

\author{
${ }^{1}$ Empa, Swiss Federal Laboratories for Materials Testing and Research, Laboratory for Functional Polymers, \\ Überlandstrasse 129, 8600 Dübendorf, Switzerland \\ 2 Paul Scherrer Institut, General Energy Research Department, 5232 Villigen PSI, Switzerland \\ ${ }^{3}$ Agency for Science, Technology and Research, Data Storage Institute, DSI Building 5, Engineering Drive 1, \\ Singapore 117608
}

\begin{abstract}
Received: 20 June 2007/Accepted: 15 October 2007
Published online: 23 November 2007 • (C) Springer-Verlag 2007

ABSTRACT The laser ablation of a photosensitive triazene polymer was investigated with a ns $\mathrm{XeCl}$ excimer laser over a broad range of thicknesses $(10-400 \mathrm{~nm})$. We found that the ablation threshold fluence increased dramatically with decreasing film thickness for films thinner than $50 \mathrm{~nm}$. Ablation on substrates with different thermal properties (sapphire, fused silica, PMMA) was investigated as well, and a clear influence of the substrate material was obtained. A mathematical model combining thermal diffusion and absorption effects was used to explain the experimental data. The model is in good agreement with the experimental data and shows that heat diffusion into the substrate plays a crucial role for the ablation process of very thin films.
\end{abstract}

PACS 52.38.Mf; 44.05.+e; 81.05.Lg

1

\section{Introduction}

Laser ablation finds a broad range of applications because it allows us to create microstructures by dry etching, deposit material from a target to a substrate, perform analysis of fragments and is also used for some special applications, such as laser plasma thrusters $[1,2]$.

Material deposition by laser ablation is a useful tool in the field of materials processing. The pulsed laser deposition (PLD) technique allows us to transfer and produce films of inorganic [3,4] and organic materials [5-8]. Matrix-assisted pulsed laser evaporation (MAPLE) can be applied to transfer small molecules from a frozen matrix to a substrate $[8,9]$. One of the most promising techniques to transfer organic thin films is laser-induced forward transfer (LIFT), which allows us to deposit and pattern materials in the same process [10-14]. It consists of the following steps: a transparent substrate is coated with the material that has to be transferred. This so-called donor substrate is placed in front of a receiver plate and a laser pulse is applied through the donor. The coated material absorbs the laser pulse and is ablatively ejected and deposited on the receiver.

Fax: +41 56310 2688, E-mail: thomas.lippert@psi.ch
This method is appropriate for metal deposition but not for sensitive materials, such as polymers, because these may be damaged by the thermal load and photochemical reactions due to irradiation. A modification of the technique can solve this problem. The principle is the application of a twolayer system: a sacrificial layer acting as an absorber and the transfer material are successively coated on the donor substrate. Upon receiving the laser pulse, the sacrificial layer absorbs the energy, is decomposed into gaseous fragments and provides the thrust for propelling the top layer onto the receiver [15]. Furthermore, the absorbing material protects the top layer from the laser pulse and is specially designed to decompose at low fluences. Among various polymers, triazenebased polymers (TP) show the lowest ablation threshold at $308 \mathrm{~nm}$ and are therefore the most promising materials for this application [16-18].

It has been shown that this sacrificial layer allows us to transfer quantum dots [19], living cells embedded in a matrix [20] and even functional pixels of organic light-emitting diodes [21].

The understanding of the ablation mechanism of the sacrificial release layer is thus of primary importance to optimize the transfer process. Laser ablation has been extensively studied, both theoretically $[22,23]$ and experimentally. Several models have been proposed for ablation of polymers, involving photochemical effects (direct bond breaking) [24-28], photothermal effects (thermally broken bonds) [29-32], photophysical (mechanical stress) [33] or a combination of them. Many investigations were published on the ablation of polymers but, up to now, mainly for thick films (micrometer range or thicker). Very few studies report the ablation of thin films (sub-micrometer to nanometer range), where the optical penetration depth and the thermal diffusion length are of the same order of magnitude as the film thickness.

This work focuses on the influence of thermal diffusion on the ablation behavior of thin films of a triazene polymer, which is particularly adapted as a sacrificial layer. The motivation is to gain knowledge of this specific system for the LIFT application. We investigated the influence of the film thickness and substrate material on the threshold fluence of triazene films upon irradiation at a wavelength of $308 \mathrm{~nm}$. We also developed a mathematical model describing the temperature distribution in the film to explain the observed effects. 
2

\section{Experimental}

The structure of the triazene polymer (TP) is shown in Fig. 1 and it was synthesized as described in [34]. Films of TP were prepared by spin coating from solutions in chlorobenzene and cyclohexanone $(1: 1 \mathrm{w} / \mathrm{w})$. Substrates for spin coating were fused silica (Suprasil 2, Heraeus, 1-mm thick), sapphire (Stecher ceramicparts $\mathrm{GmbH}$, Uetendorf, Switzerland, 1-mm thick) and polymethyl methacrylate (PMMA, Maagtechnic, Dübendorf, Switzerland, 1.2-mm thick). Ablation experiments were performed with a pulsed $\mathrm{XeCl}$ excimer laser (Compex, Lambda Physik, $t_{\mathrm{FWHM}}=$ $30 \mathrm{~ns}$ ) at $308 \mathrm{~nm}$ with a repetition rate of $2 \mathrm{~Hz}$. The fluence was adjusted with an attenuator plate. A square mask with an aperture of $2 \mathrm{~mm}$ was applied to utilize a homogeneous part of the beam, which was focused by a lens onto the sample with a magnification of 0.25 , yielding a spot size of $500 \mu \mathrm{m}$. The sample was mounted on a motorized translation stage, perpendicular to the beam and with the film facing the incident laser beam. The pulse energy at each fluence was measured by a pyroelectric energy meter (Molectron J4-09 or Gentec QE 50) placed at the end of the beam line and an average on 100 pulses was used. For measuring some energy ranges, a beam splitter was used to reduce the intensity of the beam.

The computer-controlled system allowed us to create a matrix of points for each sample, where the pulse energy and the number of pulses were varied. The ablation depth was measured by profilometry (Ambios XP-1 profilometer), allowing measurement down to $3-5 \mathrm{~nm}$. The spot size was measured by an optical microscope (Zeiss Axioplan) coupled with a digital camera (Leica DC500). Scanning electron microscopy (SEM) pictures were taken with a Philips XL30 FEG environmental scanning electron microscope device.

For each fluence, the ablation depth was plotted versus the pulse number ( 1 to 10 pulses) and the slope over the linear domain yielded the ablation rate. The latter was plotted versus the fluence, giving the characteristic ablation curve, which was fitted by the phenomenological equation

$d=\frac{1}{\alpha_{\mathrm{eff}}} \ln \frac{\Phi}{\Phi_{\mathrm{th}}}$,

where $d$ is the ablation rate, $\alpha_{\text {eff }}$ the effective absorption coefficient, $\Phi$ the fluence and $\Phi_{\text {th }}$ the threshold fluence. A second method was also used, where the ablation rate was determined by a single pulse. Rates determined as described above (multipulse) were compared with this single-pulse method and no differences were found. The second method was preferred for very thin films, where one shot was sufficient to ablate the major part of the film.

Absorption coefficients of the TP films and substrates were measured by spectrophotometry (Varian Cary 50 Scan) and the refractive index of TP was measured by ellipsometry.

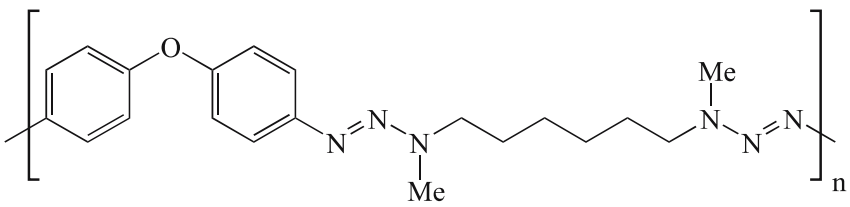

FIGURE 1 Formula of the triazene polymer
The thermal diffusivity of TP was measured with the laser flash technique (Netzsch Micro Flash) using the two-layer method: the thermal diffusivity of an aluminum plate (2-mm thick) was measured first as reference and a second time after being coated with a thick layer of TP $(110 \mu \mathrm{m})$. The thermal diffusivity of the polymer was calculated by subtracting the contribution of the aluminum plate. The heat capacity of TP was measured by differential scanning calorimetry (DSC) and a value of $1100 \mathrm{~J} / \mathrm{kg} \mathrm{K}$ at $25^{\circ} \mathrm{C}$ was obtained. For the density measurement, a piece of a thick film was floated in an aqueous $\mathrm{NaCl}$ solution by adjusting the density of the solution, which was measured at the end. A density of $1100 \mathrm{~kg} / \mathrm{m}^{3}$ was obtained by this method. The thermal conductivity was calculated from the thermal diffusivity, density and heat capacity. The physical properties of the three substrates were obtained from the literature [35-37].

\section{$3 \quad$ Mathematical model}

A mathematical model based on the linear heat transfer equations was used to compute the temperature of the film-substrate system directly after the laser pulse. The spot size is sufficiently large compared to the characteristic thermal length, allowing us to model the problem with onedimensional differential equations for the heat transfer. The indices $\mathrm{f}$ and $\mathrm{s}$ refer to film and substrate respectively and $h$ is the film thickness. The substrate thickness is $H$, which is much larger than $h$, allowing the simplification $H=\infty$. The heat-balance equations are

$\frac{1}{\chi_{\mathrm{f}}} \frac{\partial T_{\mathrm{f}}}{\partial t}=\frac{\partial^{2} T_{\mathrm{f}}}{\partial z^{2}}+Q_{\mathrm{f}}(z, t)$

for the film $0 \leq z \leq h$,

$\frac{1}{\chi_{\mathrm{s}}} \frac{\partial T_{\mathrm{s}}}{\partial t}=\frac{\partial^{2} T_{\mathrm{s}}}{\partial z^{2}}+Q_{\mathrm{s}}(z, t)$

for the substrate $h<z<\infty$.

Here $T$ is the temperature, $\chi$ the thermal diffusivity and $Q(z, t)$ describe the distribution of the absorbed laser intensity within the film and substrate. These terms can be written in the following form:

$Q_{\mathrm{f}}(z, t)=\frac{\Phi}{t_{l}} g(t) F_{\mathrm{f}}(z)$,
$Q_{\mathrm{s}}(z, t)=\frac{\Phi}{t_{l}} g(t) F_{\mathrm{s}}(z)$.

Here $\Phi$ is an average laser fluence, the $g(t)$ function describes the pulse shape:

$g(t)=\frac{t}{t_{l}} \exp \left(-\frac{t}{t_{l}}\right)$

and $t_{l}=0.409 t_{\mathrm{FWHM}}$ (the duration of the pulse at the full width at half maximum). Although it does not correspond exactly to the excimer double peak pulse shape for $308-\mathrm{nm}$ pulses, we noticed that this simplification affected only marginally the results. The function $F(z)$ in (4) and (5) represents the distri- 
bution of the laser intensity along the $z$ coordinate:

$$
\begin{aligned}
& F_{\mathrm{f}}(z)=\frac{\alpha_{\mathrm{f}}}{\kappa_{\mathrm{f}}}\left(1-R_{\mathrm{f}}\right) q_{\mathrm{f}}(z), \\
& F_{\mathrm{s}}(z)=\frac{\alpha_{\mathrm{s}}}{\kappa_{\mathrm{s}}} T_{\mathrm{s}} q_{\mathrm{s}}(z),
\end{aligned}
$$

where $\alpha$ is the absorption coefficient for the film and the substrate, $\kappa$ the thermal conductivity, $q(z)$ the normalized distribution of the laser intensity and $R_{\mathrm{f}}$ and $T_{\mathrm{S}}$ the film reflectivity and substrate transmittivity, respectively. $R_{\mathrm{f}}$ depends on the film thickness according to the following relation, valid for normal incidence [38]:

$R_{\mathrm{f}}=|r|^{2}=\left|\frac{r_{\mathrm{mf}} \mathrm{e}^{-2 \mathrm{i} \psi}+r_{\mathrm{fs}}}{\mathrm{e}^{-2 \mathrm{i} \psi}+r_{\mathrm{mf}} r_{\mathrm{fs}}}\right|^{2}$.

The index $m$ refers to the medium (air). $r_{\mathrm{mf}}$ and $r_{\mathrm{fs}}$ are the amplitude reflection coefficients on the boundaries medium/film and film/substrate respectively, given by

$r_{\mathrm{mf}}=\frac{\sqrt{\varepsilon_{\mathrm{m}}}-\sqrt{\varepsilon_{\mathrm{f}}}}{\sqrt{\varepsilon_{\mathrm{m}}}+\sqrt{\varepsilon_{\mathrm{f}}}}, \quad r_{\mathrm{fs}}=\frac{\sqrt{\varepsilon_{\mathrm{f}}}-\sqrt{\varepsilon_{\mathrm{s}}}}{\sqrt{\varepsilon_{\mathrm{f}}}+\sqrt{\varepsilon_{\mathrm{s}}}}$.

The function $\psi$ is proportional to the film thickness $h$ :

$\psi=\frac{2 \pi}{\lambda} h \sqrt{\varepsilon_{\mathrm{f}}}$,

where $\lambda$ is the laser wavelength. Thus, $R_{\mathrm{f}}$ is determined by $h$ and the three relative dielectric permittivities of the film, medium and substrate, respectively: $\varepsilon_{\mathrm{f}}, \varepsilon_{\mathrm{m}}$ and $\varepsilon_{\mathrm{s}} . \varepsilon$ is given by the refractive index $n$ and the extinction coefficient $k$, according to

$\sqrt{\varepsilon}=n+\mathrm{i} k, \quad k=\frac{\alpha \lambda}{4 \pi}$.

As the optical properties of the medium (air) are close to vacuum, we take $\varepsilon_{\mathrm{m}}=1$.

Determining $q_{\mathrm{f}}$ and $q_{\mathrm{s}}$ in (7) and (8) would require us to solve the Maxwell equations. However, for simplicity we make the following assumptions:

1. The absorption coefficient of the substrate is smaller by several orders of magnitude than that of the film. Therefore, we assume that $F_{\mathrm{s}}(z)=0$.

2. The distribution of the intensity in the film is derived from Lambert-Bouguer's law of absorption $\left(1-R_{\mathrm{f}}\right) \mathrm{e}^{-\alpha_{\mathrm{f}} z}$.

After these simplifications the initial equations (2) and (3) become

$\frac{1}{\chi_{\mathrm{f}}} \frac{\partial T_{\mathrm{f}}}{\partial t}=\frac{\partial^{2} T_{\mathrm{f}}}{\partial z^{2}}+\left(1-R_{\mathrm{f}}\right) \frac{\alpha_{\mathrm{f}}}{\kappa_{\mathrm{f}}} \frac{\Phi}{t_{l}} \frac{t}{t_{l}} \exp \left(-\frac{t}{t_{l}}\right) \mathrm{e}^{-\alpha_{\mathrm{f}} z}$,

$\frac{1}{\chi_{\mathrm{s}}} \frac{\partial T_{\mathrm{s}}}{\partial t}=\frac{\partial^{2} T_{\mathrm{s}}}{\partial z^{2}}$.

Equations (13) and (14) should be solved with boundary conditions. For the outer region we consider thermal insulation:

$\left.\kappa_{\mathrm{f}} \frac{\partial T_{\mathrm{f}}}{\partial z}\right|_{z=0}=0,\left.\quad T_{\mathrm{s}}(z)\right|_{z \rightarrow \infty}=0$ and a continuity of temperature and thermal flux between film and substrate:

$\left.T_{\mathrm{f}}\right|_{z=h}=\left.T_{\mathrm{s}}\right|_{z=h},\left.\quad \kappa_{\mathrm{f}} \frac{\partial T_{\mathrm{f}}}{\partial z}\right|_{z=h}=\left.\kappa_{\mathrm{s}} \frac{\partial T_{\mathrm{S}}}{\partial z}\right|_{z=h}$.

The initial temperatures are equal to zero:

$\left.T_{\mathrm{f}}(z)\right|_{t=0}=\left.T_{\mathrm{s}}(z)\right|_{t=0}=0$.

This problem can be solved easily by applying the Laplace transform:

$\widetilde{T}_{\mathrm{f}}(z, p)=\int_{0}^{\infty} \mathrm{e}^{-p t} T_{\mathrm{f}}(z, t) \mathrm{d} t$,

$\widetilde{T}_{\mathrm{s}}(z, p)=\int_{0}^{\infty} \mathrm{e}^{-p t} T_{\mathrm{s}}(z, t) \mathrm{d} t$.

The inverse transforms are given by

$T_{\mathrm{f}}(z, t)=\frac{1}{2 \pi \mathrm{i}} \int_{\gamma-\mathrm{i} \infty}^{\gamma+\mathrm{i} \infty} \mathrm{e}^{p t} \widetilde{T}_{\mathrm{f}}(z, p) \mathrm{d} p$,
$T_{\mathrm{s}}(z, t)=\frac{1}{2 \pi \mathrm{i}} \int_{\gamma-\mathrm{i} \infty}^{\gamma+\mathrm{i} \infty} \mathrm{e}^{p t} \widetilde{T}_{\mathrm{s}}(z, p) \mathrm{d} p$.

The Laplace transform converts (13) and (14) into a set of ordinary differential equations

$\frac{\mathrm{d}^{2} \widetilde{T}_{\mathrm{f}}}{\mathrm{d} z^{2}}-\frac{p}{\chi_{\mathrm{f}}} \widetilde{T}_{\mathrm{f}}+\frac{\left(1-R_{\mathrm{f}}\right) \Phi}{\left(1+p t_{l}\right)^{2}} \mathrm{e}^{-\alpha_{\mathrm{f}} z}=0$,

$\frac{\mathrm{d}^{2} \widetilde{T}_{\mathrm{s}}}{\mathrm{d} z^{2}}-\frac{p}{\chi_{\mathrm{s}}} \widetilde{T}_{\mathrm{s}}=0$.

These equations should be solved with the boundary conditions

$\left.\frac{\partial \widetilde{T}_{\mathrm{f}}}{\partial z}\right|_{z=0}=0,\left.\quad \widetilde{T}_{\mathrm{s}}(z)\right|_{z \rightarrow \infty}=0$,

$\left.\widetilde{T}_{\mathrm{f}}\right|_{z=h}=\left.\widetilde{T}_{\mathrm{s}}\right|_{z=h},\left.\quad \kappa_{\mathrm{f}} \frac{\partial \widetilde{T}_{\mathrm{f}}}{\partial z}\right|_{z=h}=\left.\kappa_{\mathrm{s}} \frac{\partial \widetilde{T}_{\mathrm{s}}}{\partial z}\right|_{z=h}$.

The solutions of (22) and (23) are given by

$$
\begin{aligned}
\widetilde{T}_{\mathrm{f}}= & a \exp \left(-\sqrt{\frac{p}{\chi_{\mathrm{f}}}} z\right)+b \exp \left(\sqrt{\frac{p}{\chi_{\mathrm{f}}}} z\right) \\
& -\frac{\alpha_{\mathrm{f}} \chi_{\mathrm{f}}}{\kappa_{\mathrm{f}}\left(\alpha_{\mathrm{f}}^{2} \chi_{\mathrm{f}}-p\right)} \frac{\left(1-R_{\mathrm{f}}\right) \Phi}{\left(1+p t_{l}\right)^{2}} \mathrm{e}^{-\alpha_{\mathrm{f}} z}, \\
\widetilde{T}_{\mathrm{s}}= & c \exp \left(-\sqrt{\frac{p}{\chi_{\mathrm{s}}}} z\right) .
\end{aligned}
$$

The unknown constants $a, b$ and $c$ can be obtained from the boundary conditions (24). The inverse Laplace transform is complicated to solve analytically but it can be solved numerically. The result gives the temperature of the TP film for each time and position. 


\section{General considerations}

A SEM picture of an ablation crater is shown in Fig. 2. The film is $360-\mathrm{nm}$ thick and six pulses of $90 \mathrm{~mJ} / \mathrm{cm}^{2}$ were applied. The triazene polymer exhibits a clean ablation behavior. The edges of the crater are sharp and no debris or redeposited material is visible in the vicinity of the crater. The origin of the wavy shape of the edges is unclear so far, but may arise from optical effects or from shock-wave propagation during the ablation.

The ablation rate was measured as explained above. No incubation was detected during multipulse ablation. Figure 3 shows a typical ablation curve and its corresponding fit obtained from (1). Three domains are visible: no ablation is observed below the threshold fluence; the ablation rate just above the threshold shows a logarithmic dependence on the fluence, according to (1); finally, at high enough fluences the ablation rate is limited by the film thickness. It is interesting to note that the ablation rate deviates from the fit in the range where ablation rates similar to the film thickness are predicted. In this fluence range a smooth transition to the theoretical limit (i.e. film thickness) is observed.

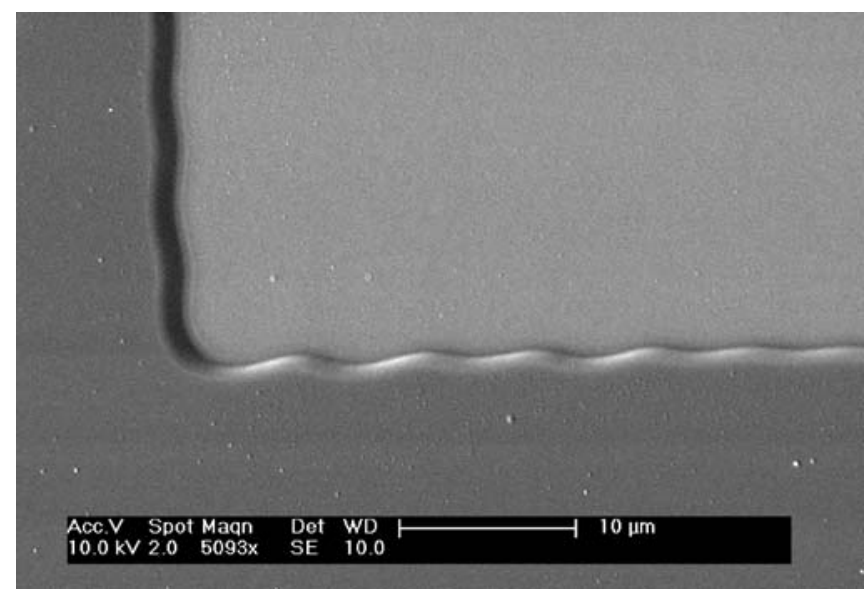

FIGURE 2 Scanning electron microscopy of an ablation crater after six pulses at $90 \mathrm{~mJ} / \mathrm{cm}^{2}$. Film thickness is $360 \mathrm{~nm}$. The sample was coated for the SEM analysis with a 3-nm layer of Pt for electrical conduction

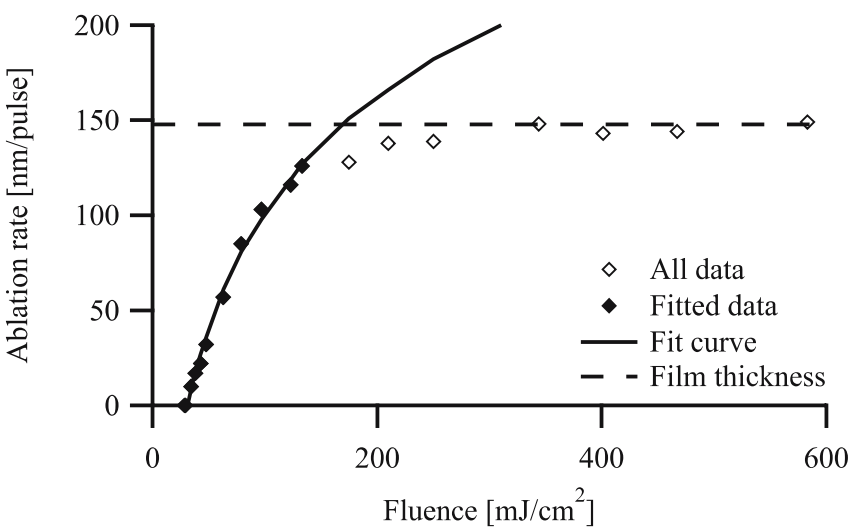

FIGURE 3 Ablation curve of TP on a fused-silica substrate. The film thick ness is $148 \mathrm{~nm}$

\section{2}

\section{Influence of the film thickness}

The influence of the film thickness on the ablation threshold is an important parameter for the application of triazene as sacrificial layer in the LIFT process. TP films with a thickness between 10 and $400 \mathrm{~nm}$ were therefore investigated in detail. Figure 4 shows the measured threshold fluences $(\bullet)$ versus film thickness for ablation experiments using fused-silica substrates. The threshold fluence is constant at 20 to $25 \mathrm{~mJ} / \mathrm{cm}^{2}$ for films thicker than $50 \mathrm{~nm}$. The absence of oscillations in the threshold fluence suggests that interference effects are not important. Below $50 \mathrm{~nm}$, a steep increase of the threshold fluence with decreasing thickness is observed. The clear influence of the film thickness on the ablation threshold of TP was a priori not expected.

To understand this phenomenon a thermal model of the evolution of the surface temperature versus film thickness was developed as described in Sect. 3. The temperature profiles for film thicknesses between 10 and $400 \mathrm{~nm}$ were computed. The applied parameters are summarized in Table 1 . The absorption coefficients at $308 \mathrm{~nm}, \alpha_{308}$, are expressed relative to the base e, consistently with (13), and a pulse duration $t_{\mathrm{FWHM}}=30 \mathrm{~ns}$ was applied. Figure 5 shows the result of the calculation as a temperature evolution of the film surface $(z=0)$ for a constant fluence $\Phi_{0}=25 \mathrm{~mJ} / \mathrm{cm}^{2}$, corresponding to the threshold fluence for thick films. Each curve corresponds to a different film thickness. It should be noted that all temperatures are expressed relatively to the initial temperature (i.e. room temperature) and are not absolute temperatures. The model allows us notably to calculate the peak temperature $T_{\max }$ reached by the film. It clearly appears that this maximum temperature of the film surface depends on the thickness. The thinner the film, the lower the peak temperature. Above $180 \mathrm{~nm}$ all temperature profiles tend to merge into each other and $T_{\max }$ approaches a constant value of $T_{0} \approx 1450 \mathrm{~K}$.

\begin{tabular}{lllll}
\hline & $\alpha_{308}\left[\mathrm{~cm}^{-1}\right]$ & $n[-]$ & $\kappa[\mathrm{W} /(\mathrm{cm} \mathrm{K})]$ & $\chi\left[\mathrm{cm}^{2} / \mathrm{s}\right]$ \\
\hline TP & $2.3 \times 10^{5}$ & 1.64 & $1.3 \times 10^{-3}$ & $1.0 \times 10^{-3}$ \\
Fused silica & $8.8 \times 10^{-1}$ & 1.49 & $1.4 \times 10^{-2}$ & $8.1 \times 10^{-3}$ \\
Sapphire & $1.7 \times 10^{0}$ & 1.81 & $3.3 \times 10^{-1}$ & $1.1 \times 10^{-1}$ \\
PMMA & $6.4 \times 10^{1}$ & 1.55 & $1.9 \times 10^{-3}$ & $1.1 \times 10^{-3}$ \\
\hline
\end{tabular}

TABLE 1 Physical properties of the TP and the substrates

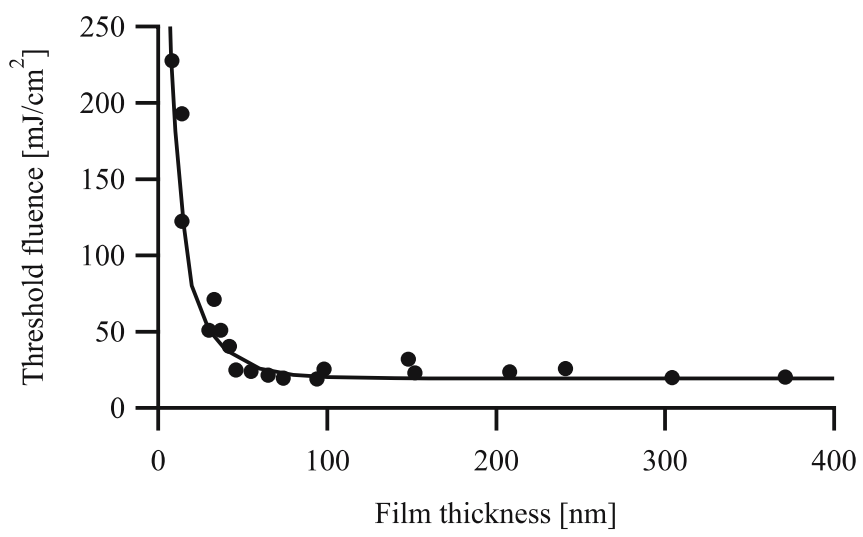

FIGURE 4 Measured (•) and calculated (solid line) threshold fluences of triazene as function of the film thickness. Substrate is fused silica 


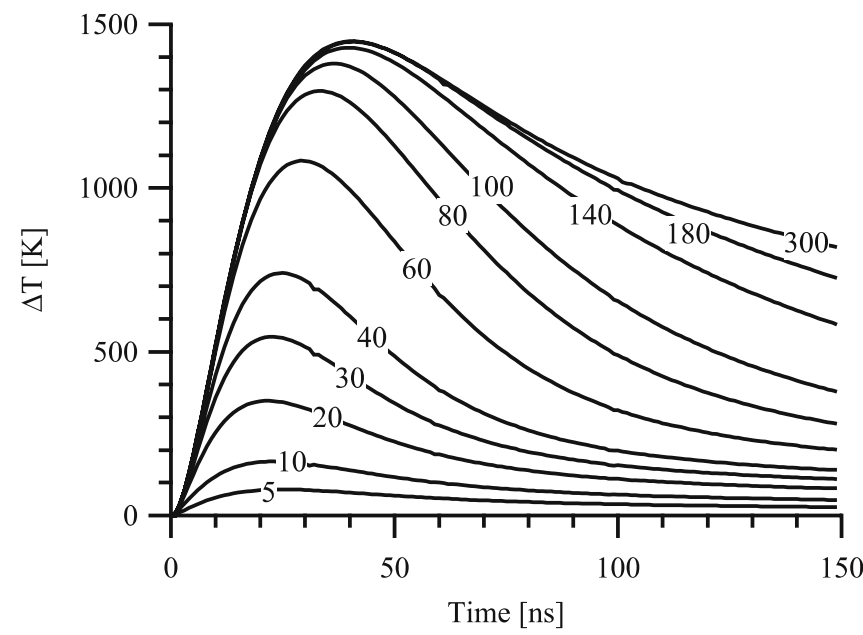

FIGURE 5 Evolution of the film surface temperature for different thicknesses of film ( $h[\mathrm{~nm}]$ written on each curve) at a constant fluence of $25 \mathrm{~mJ} / \mathrm{cm}^{2}$. Temperature is given relative to room temperature. Substrate is fused silica

Now, for films thinner than $60-80 \mathrm{~nm}$, no ablation occurs at $25 \mathrm{~mJ} / \mathrm{cm}^{2}$, contrary to thicker films. There is thus an obvious relationship between the peak temperature and the ablation onset. To quantify it, we consider that a certain temperature is needed for ablation to start. This temperature is defined as $T_{\text {th }}$ and depends on both fluence and film thickness. For films thicker than $180 \mathrm{~nm}$, this threshold temperature is $T_{0}$, which corresponds to the observed constant threshold fluence, while for thinner films a higher fluence is needed to reach $T_{0}$.

Using the model, we recalculate the temperature profiles for each $h$ by varying $\Phi$. The fluence needed to reach $T_{0}$ is then the threshold fluence $\Phi_{\text {th }}$, which satisfies the relation

$T_{\max }\left(h, \Phi_{\text {th }}\right)=T_{0}$

The results of this calculation are shown in Fig. 4 (solid line) together with the experimental data points. The model correlates very well with the experiment. A significant change in the calculated threshold appears below $100 \mathrm{~nm}$. This originates from two effects. Firstly, the diminution of the absorbed photons starts to affect the ablation below the optical distance $h_{\mathrm{opt}} \approx \alpha_{\mathrm{eff}}^{-1}$. In this case, $h_{\mathrm{opt}}$ is $45 \mathrm{~nm}$. The second effect is due to the large differences in thermal conductivities between the substrate and the film. Below the thermal diffusion length $h_{\mathrm{th}} \approx \sqrt{2 \chi_{\mathrm{f}} t_{l}}=85 \mathrm{~nm}$, the films start to be thermally influenced by the substrate. In the case of a better conducting substrate, it acts a heat sink and a higher fluence is needed for the film to reach the threshold temperature.

Returning to Fig. 3, the smooth transition from the fitted curve to the theoretical limit can now be explained. The closer to the substrate, the better the heat is transferred. Therefore, compared to the bulk polymer, the layer close to the substrate needs to receive more heat to reach the threshold temperature and undergo ablation. As shown in Fig. 4, the threshold fluence rises abruptly with decreasing thickness, thus explaining the asymptotic transition in Fig. 3.

It has to be pointed out that our thermal model does not account for all possible terms in the heat balance. In particular, the exothermic decomposition of the triazene leads to a temperature increase in the order of several hundreds of $\mathrm{K}$ and heat removal may occur by fragments. These effects become important for a significant ablation depth. Considering the threshold fluence, we are at the point where the ablation depth is virtually zero, meaning that the decomposed amount of material is very low. If we compare the decomposition energy of a very thin layer with the energy provided by the laser pulse, the ratio converges to zero when the fluence becomes closer to the threshold. Therefore, the decomposition energy can be neglected for predicting the threshold fluence. This explains why, despite these simplifications, the model describes the experimental behavior very well.

\subsection{Influence of the substrate material}

A detailed knowledge of the ablation behavior with other substrates can give very useful information for two aspects. The validity of the model can be checked, which predicts an influence of the thermal conductivity of the substrate on the threshold fluence. The other aspect is related to the envisioned application, i.e. LIFT. It will be advantageous for certain processes to use a polymeric material as substrate instead of fused silica. The influence of the substrate on the threshold fluence was therefore investigated with two other materials. Sapphire was chosen as a highly conductive material and PMMA as a low conductor. For the latter, the ablation threshold is high enough $\left(900-1100 \mathrm{~mJ} / \mathrm{cm}^{2}[39,40]\right)$ to ensure that the substrate does not undergo ablation in the investigated fluence range.

The influence of the substrate material on the threshold fluence is presented in Fig. 6. The dots are the measured points while the lines represent the values calculated with the model, using the parameters in Table 1. The same effect of the thickness is observed for the two other substrates but, as predicted, with different magnitudes which depend on the material. Ablation on sapphire exhibits the strongest thickness-related effect, followed by fused silica, while the polymeric substrate (PMMA) has the lowest influence on the threshold fluence. The error in the fluence and thickness measurements is estimated to be $10 \%$. Almost all experimental points fit the

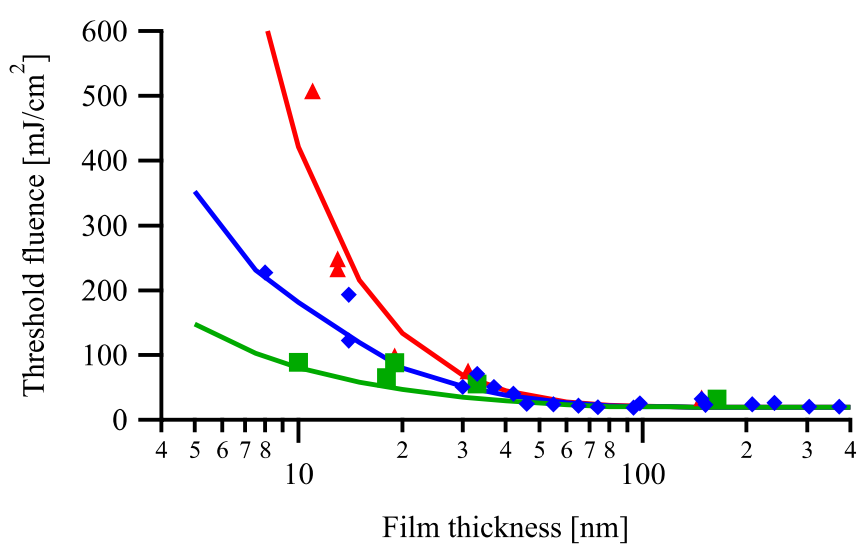

FIGURE 6 Influence of the substrate material on the ablation threshold. The points are experimental data: $(\boldsymbol{\Delta})$ sapphire, $(\boldsymbol{\bullet})$ fused silica, ( $\mathbf{\square})$ PMMA. The solid lines are computed from the model: (up) sapphire, (middle) fused silica, (down) PMMA 
corresponding curve within the uncertainty. Although some points still deviate, the trend is a clear difference between the substrates.

The experiments confirm the validity of the model. The substrate with the higher thermal conductivity will transfer more heat and thus reduce the maximum temperature reached at the film surface. On the other hand, PMMA has a thermal conductivity similar to TP and thus acts thermally as an extension of the TP layer. In this case the increase of the threshold fluence is mainly due to the reduced amount of photons absorbed within the small thickness, as reported by Daminelli et al. [41] for the ablation of hydrogenated carbon films coated on an insulating substrate. It appears from Table 1 that the absorption coefficient of PMMA is higher than for sapphire and fused silica. One could argue that it is not clear whether the lower thermal conductivity or the higher absorption is responsible for the observed effect. Considering that the absorption coefficient of PMMA is more than a thousand times lower than that of TP, the fluence absorbed by the substrate remains negligible.

\subsection{Considerations on the mechanism}

This work shows that the thermal contribution plays an important role in the ablation of very thin triazene films, as discussed previously for thick samples [17, 42, 43]. However, it is difficult to conclude the ablation mechanism from these experiments. As a matter of fact, we focused specifically on very thin films and showed that their threshold fluence is higher than for bulk polymer, originating from thermal diffusion and a laser penetration depth larger than the film thickness. For thin films, it is possible to prove the thermal contribution in the ablation process, which is more difficult in bulk material. It may therefore not be feasible to extrapolate a general ablation mechanism from the observations on very thin films. Moreover, the mechanism consists of a mixture of thermal degradation, photochemical and thermally activated photochemical bond breaking, which cannot be quantified with the analysis of the threshold fluence and ablation rate. The nature of the mechanism cannot be assessed without a detailed analysis of composition of the ablation products [24]. Additionally, it has been shown that a switch from a photochemical to a photothermal mechanism can happen with increasing laser fluence $[22,44,45]$. For these reasons, we believe that a definitive conclusion on the mechanism cannot be drawn from this work.

\section{5}

\section{Conclusions}

The triazene polymer is a suitable material for LIFT applications due to the clean ablation behavior and low threshold fluence. For film thicknesses below $50 \mathrm{~nm}$ a pronounced change of the threshold fluence is observed. The developed model describes the experimental data very well, showing that the thermal diffusion into the substrate and the reduced number of absorbed photons are responsible for the increase of the threshold fluence for low film thicknesses. The validity of the model has been confirmed by changing the substrate material, where a strong effect related to the thermal conductivity of the substrate is demonstrated. These results reveal an important aspect of the laser ablation of polymers and have a large implication in the processing or application of thin films.

ACKNOWLEDGEMENTS Financial support from the Swiss National Science Foundation is gratefully acknowledged. We would also like to thank Philippe Gasser for the SEM pictures, Pascal Feurer for help with sample preparation, Laura Bocher for assistance with thermal conductivity measurements and Rüdiger Kötz for the ellipsometry measurement.

\section{REFERENCES}

1 D. Bäuerle, Laser Processing and Chemistry, 3rd edn. (Springer, Berlin, 2000)

2 L. Urech, M. Hauer, T. Lippert, C. Phipps, E. Schmid, A. Wokaun, I. Wysong, Proc. SPIE 5448, 52 (2004)

3 V.S. Ban, D.A. Kramer, J. Mater. Sci. 5, 978 (1970)

4 S. Hansen, T. Robitaille, Appl. Phys. Lett. 50, 359 (1987)

5 N. Matsumoto, H. Shima, T. Fujii, F. Kannari, Appl. Phys. Lett. 71, 2469 (1997)

6 X. Yang, Y. Tang, M. Yu, Q. Qin, Thin Solid Films 358, 187 (2000)

7 G. Blanchet, Macromolecules 28, 4603 (1995)

8 D. Chrisey, A. Piqué, R. McGill, J. Horwitz, B. Ringeisen, D. Bubb, P. Wu, Chem. Rev. 103, 553 (2003)

9 D. Chrisey, A. Piqué, J. Fitz-Gerald, R. Auyeung, R. McGill, H. Wu, M. Duignan, Appl. Surf. Sci. 154-155, 593 (2000)

10 J. Bohandy, B.F. Kim, F.J. Adrian, J. Appl. Phys. 60, 1538 (1986)

11 C. Arnold, P. Serra, A. Piqué, MRS Bull. 32, 23 (2007)

12 K. Kyrkis, A. Andreadaki, D. Papazoglou, I. Zergioti, in Recent Advances in Laser Processing of Materials, ed. by J. Perrière, E. Millon, E. Fogarassy (Elsevier, Amsterdam, 2006), pp. 213-241

13 I. Zergioti, S. Mailis, N. Vainos, C. Fotakis, S. Chen, C. Grigoropoulos, Appl. Surf. Sci. 127-129, 601 (1998)

14 H. Esrom, J. Zhang, U. Kogelschatz, A. Pedraza, Appl. Surf. Sci. 86, 202 (1995)

15 T. Mito, T. Tsujita, H. Masuhara, N. Hayashi, K. Suzuki, Japan. J. Appl. Phys. Part 2 40, L805 (2001)

16 D. Karnakis, T. Lippert, N. Ichinose, S. Kawanishi, H. Fukumura, Appl. Surf. Sci. 127-129, 781 (1998)

17 T. Lippert, J. Dickinson, Chem. Rev. 103, 453 (2003)

18 T. Lippert, Adv. Polym. Sci. 168, 51 (2004)

19 J. Xu, J. Liu, D. Cui, M. Gerhold, A.Y. Wang, M. Nagel, T.K. Lippert, Nanotechnology 18, 025403 (2007)

20 A. Doraiswamy, R. Narayan, T. Lippert, L. Urech, A. Wokaun, M. Nagel, B. Hopp, M. Dinescu, R. Modi, R. Auyeung, D. Chrisey, Appl. Surf. Sci. 252, 4743 (2006)

21 R. Fardel, M. Nagel, F. Nüesch, T. Lippert, A. Wokaun, Appl. Phys. Lett. 91, 061103 (2007)

22 N.M. Bityurin, B. Luk'yanchuk, M. Hong, T. Chong, Chem. Rev. 103, 519 (2003)

23 S. Anisimov, B. Luk'yanchuk, Phys. Uspekhi 45, 293 (2002)

24 M. Prasad, P.F. Conforti, B.J. Garisson, J. Appl. Phys. 101, 103113 (2007)

25 N. Mansour, K. Jamshidi-Ghaleh, J. Phys. D 38, 852 (2005)

26 M. Castex, N.M. Bityurin, Appl. Surf. Sci. 197-198, 805 (2002)

27 H. Schmidt, J. Ihlemann, B. Wolff-Rottke, K. Luther, J. Troe, J. Appl. Phys. 83, 5458 (1998)

28 G. Pettit, R. Sauerbrey, Appl. Phys. A 56, 51 (1993)

29 G. Bounos, A. Selimis, S. Georgiou, E. Rebollar, M. Castillejo, N.M. Bityurin, J. Appl. Phys. 100, 114323 (2006)

30 N.M. Bityurin, Proc. SPIE 4423, 197 (2001)

31 N. Arnold, N.M. Bityurin, D. Bäuerle, Appl. Surf. Sci. 138-139, 212 (1999)

32 N.M. Bityurin, N. Arnold, B. Luk'yanchuk, D. Bäuerle, Appl. Surf. Sci. 127-129, 164 (1998)

33 B. Luk'yanchuk, N.M. Bityurin, S. Anisimov, A.Y. Malyshev, N. Arnold, D. Bäuerle, Appl. Surf. Sci. 106, 120 (1996)

34 M. Nagel, R. Hany, T. Lippert, M. Molberg, F. Nüesch, D. Rentsch, Macromol. Chem. Phys. 208, 277 (2007)

35 J. Brandrup, E. Immergut, E. Grulke, Polymer Handbook, 4th edn. (Wiley-Interscience, New York, 1999)

36 D.R. Lide, CRC Handbook of Chemistry and Physics, 87th edn. (Taylor and Francis, New York, 2006)

37 Heraeus Quartzglas GmbH, Suprasil 2 product data sheet, Tech. Rep., www.heraeus-quarzglas.com (1994) 
38 L. Landau, E. Lifshitz, Electrodynamics of Continuous Media (Butterworth-Heinemann, Oxford, 1984)

39 R. Srinivasan, E. Sutcliffe, B. Braren, Appl. Phys. Lett. 51, 1285 (1987)

40 A. Costela, J.M. Figuera, F. Florido, I. García-Moreno, E.P. Collar, R. Sastre, Appl. Phys. A 60, 261 (1995)

41 G. Daminelli, S. Pentzien, A. Hertwig, J. Krüger, Appl. Phys. A 83, 89 (2005)
42 T. Lippert, S. Langford, A. Wokaun, S. Georgiou, J. Dickinson, J. Appl. Phys. 86, 7116 (1999)

43 T. Lippert, L. Bennett, T. Nakamura, H. Niino, A. Ouchi, A. Yabe, Appl. Phys. A 63, 257 (1996)

44 N.M. Bityurin, Annu. Rep. Prog. Chem. C 101, 216 (2005)

45 K. Hatanaka, M. Kawao, Y. Tsuboi, H. Fukumura, J. Appl. Phys. 82, 5799 (1997) 